(2) Open Access Full Text Article

\title{
Expanding the National Drug Abuse Treatment Clinical Trials Network to address the management of substance use disorders in general medical settings
}

\author{
This article was published in the following Dove Press journal: \\ Substance Abuse and Rehabilitation \\ 23 July 2014 \\ Number of times this article has been viewed
}

\section{Betty Tai \\ Steven Sparenborg \\ Udi E Ghitza \\ David Liu \\ Center for the Clinical Trials Network, National Institute on Drug Abuse, National Institutes of Health, Bethesda, Maryland, USA}

Correspondence: Betty Tai Center for the Clinical Trials Network, National Institute on Drug Abuse, 600I Executive Blvd, Room 3I20, Bethesda, Maryland, 20892, USA

Tel +I 30I 4436697

Email btai@nih.gov

\begin{abstract}
The Patient Protection and Affordable Care Act (2010) and the Mental Health Parity and Addiction Equity Act (2008) expand substance use disorder (SUD) care services in the USA into general medical settings. Care offered in these settings will engage substance-using patients in an integrated and patient-centered environment that addresses physical and mental health comorbidities and follows a chronic care model. This expansion of SUD services presents a great need for evidence-based practices useful in general medical settings, and reveals several research gaps to be addressed. The National Drug Abuse Treatment Clinical Trials Network of the National Institute on Drug Abuse can serve an important role in this endeavor. High-priority research gaps are highlighted in this commentary. A discussion follows on how the National Drug Abuse Treatment Clinical Trials Network can transform to address changing patterns in SUD care to efficiently generate evidence to guide SUD treatment practice within the context of recent US health care legislation.
\end{abstract}

Keywords: Patient Protection and Affordable Care Act, National Drug Abuse Treatment Clinical Trials Network, substance use disorders, practice-based research network, electronic health records

\section{Introduction}

The Patient Protection and Affordable Care Act (2010) and other recent federal health care legislation are bringing about substantial changes in how the US health care system provides treatment for substance use disorders (SUD) to those who need it. ${ }^{1}$ Mental health and/or SUD services benefits are expanding to include approximately 32 million formerly uninsured people. ${ }^{2}$ The Patient Protection and Affordable Care Act promotes the integration of a wide array of medical services, including behavioral health care, into patient-centered medical homes, health maintenance organizations, or accountable care organizations, which are typically affiliated with local health departments and/or community health centers. ${ }^{3}$ The Mental Health Parity and Addiction Equity Act (2008) mandates group health plans and insurers to offer health care benefits for SUD treatment services that are comparable with coverage for general medical and surgical care. ${ }^{1,2}$ Consequently, many health plans are expected to offer SUD prevention, early intervention, and treatment services in integrated treatment systems. ${ }^{4-6}$ Taken together, these changes in the health care landscape are bringing SUD care into general medical settings and into better alignment with the management of other chronic illnesses. ${ }^{4-6}$ 
Another recently enacted piece of legislation, the Health Information Technology for Economic and Clinical Health Act (2009), is stimulating the adoption of electronic health record (EHR) systems and the meaningful use of fully certified health information technology. ${ }^{7}$ This legislative mandate has accelerated the development and implementation of interoperable EHR systems, which enable the free flow of patients' health-related information among various medical practices and facilities and the provision of coordinated, patient-centered comprehensive care. ${ }^{8-10}$

In summary, recently enacted legislation is effecting profound changes in how, where, and to whom SUD care is delivered. ${ }^{1-7}$ The number of patients with unhealthy substance use behaviors or a SUD that can benefit from health care could increase more than ten-fold. ${ }^{2}$ The services available to them will expand from specialty SUD or mental health care facilities for treatment seekers to general medical settings. This expansion should facilitate the adoption of chronic care models for the management of substance use problems and the integration of these services into the mainstream of the health care system..$^{3-5,11}$

\section{Research to guide expanded SUD care in general medical settings}

The unprecedented expansion of health care engagement and utilization by SUD patients calls for new research to guide evidence-based practices for SUD care in general medical settings. There are several key research gaps that need to be addressed; existing clinical research programs, such as the National Drug Abuse Treatment Clinical Trials Network (CTN) at the National Institute on Drug Abuse (NIDA), can serve an important role in this endeavor. The discussion that follows enumerates the research gaps and then suggests how the CTN can be reshaped to address changing patterns in SUD care and efficiently conduct research that can guide SUD treatment practice.

\section{SUD detection and patient engagement in general medical settings}

Engaging primary care providers to undertake routine or targeted SUD screening and assessment of their patients is the first critical step in integrating SUD care into general medical settings. Screening and assessment offer the promise of early detection of patients' unhealthy substance use, prompting measures to prevent progression to a fully developed addiction. Screening and assessment leading to appropriate and timely care may be of particular benefit to high-risk patient populations, ${ }^{10}$ including young adults and adolescents. ${ }^{12}$
For patients with co-occurring chronic conditions, identifying and addressing substance use problems may improve health care outcomes and quality of life. ${ }^{6,11,13}$ Research in this area can inform the reviews and practice recommendations of the US Preventive Services Task Force and, more generally, support health care providers' adoption of effective screening, assessment, and treatment methods. To establish the necessary evidence of effectiveness to guide the identification and management of SUDs in general medical settings, research to develop and evaluate the following is needed: ${ }^{5}$

- Validated, brief, and actionable SUD screening and assessment tools that are suitable for efficient and userfriendly administration in general medical settings

- Evidence-based brief interventions for SUDs that can be efficiently integrated into the busy workflows of primary care settings and tailored to match the level of risk identified through the brief assessment tools

- Evidence-based, sustainable, and scalable implementation methods to engage and train primary care providers to adopt effective screening and assessment tools and interventions in their practices

- Practical approaches to effectively build linkage and referral techniques between primary care and SUD specialty care and leverage mobile technologies and telemedicine to enhance the reach of these techniques

- Outcome measures, such as rates of hospital readmission and emergency department visits or measures of improvement or progression of co-occurring illnesses, that may be of particular relevance for patients and providers in general medical settings

- Clinical quality measures to substantiate the research evidence and to facilitate policy development to promote the adoption of screening and assessment tools and treatment interventions in general medical settings. ${ }^{5}$

\section{Chronic care models for management of SUDs}

SUDs are chronic, relapsing brain diseases, ${ }^{14}$ yet current treatment regimens for SUDs largely follow an acute disease care model. ${ }^{4}$ Research on SUD treatment has largely been focused on care provided in specialty care settings for a short duration or in an episodic fashion; ${ }^{4}$ there is a lack of research evidence to guide strategies for treating SUD patients under a chronic care model of disease management., ${ }^{5,11}$

In order to fill this gap, the development of patient registries for SUDs that enroll participants from general medical settings and/or communities is needed. ${ }^{5,15-17}$ With the widespread adoption of EHRs by clinical providers, patient 
registries that draw upon patients' personal EHR data will allow for comprehensive longitudinal data collection on the natural history of SUD progression, treatment utilization, and outcomes. Systematically collected longitudinal data may shed light on how, when, and why patients relapse and seek treatment. This information is essential to the task of devising effective chronic care algorithms to prevent drug use relapse, promote recovery, and maintain good health behaviors. Patient registry infrastructures may also be used as a platform for conducting clinical trials in SUDs, facilitating the accelerated recruitment of community residents into trials, streamlining of data collection and follow-up procedures, and evaluation of health status and health care utilization outcome measures. ${ }^{5,14}$ Trials that utilize registries in this way can achieve savings in both time and costs. ${ }^{18,19}$

\section{Care for patients with multiple chronic co-occurring conditions}

It is estimated that one in four Americans, and more than half of those who are 65 years of age and older, suffer from more than one chronic medical condition. ${ }^{20,21}$ For many, one of these conditions is a SUD. There is a critical need to understand better how co-occurring conditions, such as major depression, diabetes, asthma, cancers, and cardiovascular disease, are related to the severity of SUD conditions and SUD treatment outcomes. Integrated health care systems, development of which is promoted by recent US federal health care legislation, provide natural settings and rationales for research to investigate these relationships and strategies for addressing them.

It has been well recognized that the randomized controlled trial is the gold standard for establishing evidencebased medical practices, but randomized controlled trials are often very costly, complex in design and execution, and slow to produce research results. Confronting these shortcomings, the US Institute of Medicine convened roundtable discussions with stakeholders, including patients, providers, and researchers with diverse perspectives and technical expertise, and recommended the development of a "continuously learning health care system" that integrates research and clinical practice. $^{22}$ In a learning health care system, generation of practice knowledge is embedded in the core of the practice of medicine. The evidence generated is then a natural outgrowth of the health care delivery process and leads to continual improvement in care. ${ }^{23}$

With the wide adoption of EHRs by health care providers, EHRs have the potential to become the primary vehicle for capturing clinical research data and are therefore a central element in the implementation of a learning health care system. Realizing this potential will require investments in the development of new technologies and methods for using EHRs in clinical research, a common data infrastructure to facilitate compilation and analysis of data from various sources, and new regulatory rules and policies to guide the proper use of clinical records for research purposes. ${ }^{22}$

The learning health care system approach promotes the integration of research and practice and moves research into clinical practice settings; the engagement of providers and patients in all phases of the research cycle is critical. In such a model, clinical study results generated in the health care system can lead directly to changes in clinical practice and improved patient care. Progress towards this end will require a major transformation of the clinical research enterprise, encompassing its infrastructure, workforce, research settings, data collection and management methods, and study design and procedures. ${ }^{21,22}$

\section{Transforming the CTN}

NIDA's CTN is built upon collaboration between clinical researchers and treatment providers, and since its inception, the network has sought to design and conduct research that can directly inform SUD treatment practice. While the CTN has undertaken research projects on SUD care in general medical settings in the USA, ${ }^{24}$ to this point the network's efforts have primarily been focused on treatment provided in specialty care settings. In light of the accelerating integration of SUD care into the medical mainstream, and the consequent demand for research evidence to guide the delivery of SUD care in these contexts, the CTN must address the critical knowledge gaps outlined in the preceding sections of this commentary. To do so effectively and efficiently will require a considered reshaping of the CTN clinical research enterprise. The transformation will include modifications of the network's infrastructure to engage general medical providers as partners in research, the adoption of new research strategies, including point-of-care approaches, ${ }^{25}$ and the development of new methods and tools for SUD data collection and data sharing.

\section{Expanding the network}

The CTN must expand its research infrastructure to engage general health care provider organizations, such as federally qualified health centers, accountable care organizations, patient-centered medical homes, and health maintenance organizations, and ideally practice-based research networks 
that are built upon such organizations. ${ }^{16}$ This infrastructure expansion is necessary to allow the CTN to efficiently engage stakeholders from general medical settings to be its research partners. If digitized SUD patient registries can be established in existing health care networks, then it should be possible to use the registries as a platform to conduct cost-efficient clinical trials, as experiences in other fields of medical research have demonstrated. ${ }^{18,19}$ Such randomized registry trials and, more generally, the expansion of SUD research from specialty care settings into general medical settings, will also broadly improve the external validity of clinical trial results and facilitate the adoption of new prevention and intervention practices by primary care providers.

One example of a practice-based research network is the PCORnet, the National Patient-Centered Clinical Research Network. The PCORnet, recently established by the US Patient-Centered Outcomes Research Institute, is a large, national network that supports clinical outcomes research efforts. Through PCORnet, the US Patient-Centered Outcomes Research Institute aims to foster a range of observational and experimental comparative effectiveness research endeavors by establishing a resource of clinical data gathered in "real-time" and in "real-world" settings. ${ }^{26}$ Collaborations between the CTN and established networks like PCORnet will allow for a synergy of resources and create opportunities for novel, pragmatic, and cost-effective SUD clinical research projects.

\section{Developing new methods for data collection and data sharing}

The use of digital data systems to integrate information across all aspects of health care and biomedical research has the potential to transform health care delivery and clinical research. ${ }^{27}$ As noted above, EHRs could become the principal vehicle for capturing clinical research data. This prospect entails both potential benefits and pitfalls. The promise of producing readily generalizable research results is one key advantage; EHR data from general medical settings identify a comprehensive demographic spectrum of patients, including patients with complex "real-world" arrays of co-occurring conditions. The very large number of patients that can be aggregated through EHRs will enable researchers to correlate genotypic data with clinical phenotypic data and accelerate "big data" analysis to inform personalized medical care. Moreover, the potential power of sharing large, interoperable databases for secondary data use cannot be overstated. ${ }^{28}$

However, the utilization of EHRs in research poses several important challenges; first among them is the need to achieve wide acceptance of uniform data domains, vocabulary, standards, and interoperability. Several steps must be considered to enable the use of EHRs to facilitate SUD clinical research in particular. First, there is a need to integrate SUD measurements into EHRs and to encourage the routine collection of key SUD data as part of usual care in general medical settings. ${ }^{9}$ With this objective in mind, NIDA, through the CTN, is striving to develop common data elements of SUD domains for both EHRs and SUD research. ${ }^{29}$ This initiative aims to establish a basis (eg, screening and assessment items for substance use) for SUD care to be integrated into primary care practice and to facilitate the use of new EHR-based data resources in clinical research. ${ }^{29}$

Patient privacy protections are also of critical importance for any research endeavor that seeks to use data drawn from clinical sources. Researchers and institutional review boards must ensure that appropriate procedures for informed consent and, for secondary uses of data, de-identification and data aggregation, are undertaken. In some circumstances, SUD clinical researchers must take into account the special privacy provisions afforded to SUD patient records by Title 42, Part 2, of the US Code of Federal Regulations. ${ }^{15}$

Together with the formation and expansion of practicebased research networks, the wide adoption of health information technologies in clinical practice offers an opportunity to integrate clinical trials into clinical care delivery systems. ${ }^{21}$ The CTN, and the field of SUD clinical research in general, should continue to explore every means of taking up this opportunity to develop resources and infrastructures to support cost-efficient, pragmatic SUD trials, with the aim of accelerating the translation of effective practices from research into clinical care.

\section{Conclusion}

Recent US federal health care legislation, including the Patient Protection and Affordable Care Act, Mental Health Parity and Addiction Equity Act, and Health Information Technology for Economic and Clinical Health Act, is spurring transformative changes in the provision of SUD care. ${ }^{1-7}$ The expansion of health care insurance coverage and the augmentation of coverage mandates for SUD care are making treatment for SUDs and co-occurring conditions newly accessible to millions of patients and prompting the expansion of SUD screening, assessment, and treatment services into general medical settings. ${ }^{2,5}$ When the legislative vision is fully realized, patients presenting for primary care with multiple chronic co-occurring conditions, including SUDs, should expect to receive more integrated care, ideally 
coordinated within a chronic care or patient-centered medical home model. ${ }^{1,5,6}$ However, there is an urgent need for research evidence to guide the implementation of this new paradigm for SUD care.

To meet the challenge of filling the critical research gaps, the SUD clinical research community and, in particular, the NIDA CTN, should: expand clinical research infrastructures to include practice-based research networks in general medical settings; develop the "learning health care system" model for conducting practical trials ${ }^{30}$ and comparative effectiveness research; ${ }^{31}$ identify and evaluate feasible chronic care models for the treatment of SUDs and co-occurring conditions; ${ }^{11}$ and leverage modern health information technology, including EHRs, as new resources for data collection and data sharing. ${ }^{5,8-10,29}$ These foundational steps should help to ensure that new models for mainstreamed, integrated SUD care can be built upon a sturdy base of research evidence.

\section{Acknowledgments}

BT, SS, UEG, and DL are employees of the Center for the Clinical Trials Network, National Institute on Drug Abuse, National Institutes of Health, which is the funding agency for the National Drug Abuse Treatment Clinical Trials Network. The opinions in this paper are those of the authors and do not represent the official position of the US government.

\section{Disclosure}

The authors report no conflicts of interest in this work.

\section{References}

1. Buck JA. The looming expansion and transformation of public substance abuse treatment under the Affordable Care Act. Health Aff (Millwood). 2011;30:1402-1410.

2. Beronio K, Po R, Skopec L, Glied S. Affordable Care Act expands mental health and substance use disorder benefits and federal parity protections for 62 million Americans. Washington, DC, USA: Department of Health and Human Services, Office of the Assistant Secretary for Planning and Evaluation, 2013. (ASPE Issue Brief). Available from: http://aspe.hhs. gov/health/reports/2013/mental/rb_mental.cfm. Accessed April 14, 2014.

3. US Department of Health and Human Services. Read the Law: The Affordable Care Act, Section by Section. Available from: http://www. healthcare.gov/law/full/index.html. Accessed April 14, 2014.

4. Tai B, Volkow ND. Treatment for substance use disorder: opportunities and challenges under the affordable care act. Soc Work Public Health. 2013;28:165-174.

5. Ghitza UE, Tai B. Challenges and opportunities for integrating preventive substance-use-care services in primary care through the Affordable Care Act. J Health Care Poor Underserved. 2014;25(Suppl 1):36-45.

6. McLellan AT, Woodworth AM. The Affordable Care Act and treatment for "substance use disorders:" implications of ending segregated behavioral healthcare. J Subst Abuse Treat. 2014;46:541-545.

7. Buntin MB, Jain SH, Blumenthal D. Health information technology: laying the infrastructure for national health reform. Health Aff (Millwood). 2010;29:1214-1219.
8. Tai B, McLellan AT. Integrating information on substance use disorders into electronic health record systems. J Subst Abuse Treat. 2012;43: 12-19.

9. Ghitza UE, Sparenborg S, Tai B. Improving drug abuse treatment delivery through adoption of harmonized electronic health record systems. Subst Abuse Rehabil. 2011;2:125-131.

10. Tai B, Wu LT, Clark HW. Electronic health records: essential tools in integrating substance abuse treatment with primary care. Subst Abuse Rehabil. 2012;3:1-8.

11. McLellan AT, Starrels JL, Tai B, et al. Can substance use disorders be managed using the chronic care model? Review and recommendations from a NIDA Consensus Group. Public Health Rev. In press 2014.

12. Committee on Substance Abuse, Levy SJ, Kokotailo PK. Substance use screening, brief intervention, and referral to treatment for pediatricians. Pediatrics. 2011;128:e1330-e1340.

13. Ghitza UE, Wu LT, Tai B. Integrating substance abuse care with community diabetes care: implications for research and clinical practice. Subst Abuse Rehabil. 2013;4:3-10.

14. Volkow ND, Li TK. The neuroscience of addiction. Nat Neurosci. 2005;8:1429-1430.

15. Hu LL, Sparenborg S, Tai B. Privacy protection for patients with substance use problems. Subst Abuse Rehabil. 2011;2:227-233.

16. Bhattacharya S, Dunham AA, Cornish MA, et al. The measurement to understand reclassification of disease of Cabarrus/Kannapolis (MURDOCK) study community registry and repository. Am J Transl Res. 2012;4:458-470.

17. Tenenbaum JD, Christian V, Cornish MA, et al. The MURDOCK study: a long-term initiative for disease reclassification through advanced biomarker discovery and integration with electronic health records. Am J Transl Res. 2012;4:291-301.

18. Lauer MS, D'Agostino RB Sr. The randomized-registry trial - the next disruptive technology in clinical research? N Engl J Med. 2013;369: 1579-1581.

19. Fröbert O, Lagerqvist B, Olivecrona GK, et al; TASTE Trial. Thrombus aspiration during ST-segment elevation myocardial infarction. $N$ Engl J Med. 2013;369:1587-1597.

20. US Department of Health and Human Services. Multiple chronic conditions - a strategic framework: optimum health and quality of life for individuals with multiple chronic conditions. Washington, DC, USA US Department of Health and Human Services; 2010. Available from: http://www.hhs.gov/ash/initiatives/mcc/mcc_framework.pdf. Accessed April 14, 2014.

21. Institute of Medicine. Envisioning a Transformed Clinical Trials Enterprise in the United States: Establishing an Agenda for 2020. Workshop Summary. Washington, DC, USA: National Academies Press; 2012.

22. Institute of Medicine. Best Care at Lower Cost: The Path to Continuously Learning Health Care in America. Washington, DC, USA: National Academies Press; 2012.

23. Green LW. Public health asks of systems science: to advance our evidence-based practice, can you help us get more practice-based evidence? Am J Public Health. 2006;96:406-409.

24. Donovan DM, Bogenschutz MP, Perl H, et al. Study design to examine the potential role of assessment reactivity in the Screening, Motivational Assessment, Referral, and Treatment in Emergency Departments (SMART-ED) protocol. Addict Sci Clin Pract. 2012;7:16.

25. Fiore LD, Brophy M, Ferguson RE, et al. A point-of-care clinical trial comparing insulin administered using a sliding scale versus a weightbased regimen. Clin Trials. 2011;8:183-195.

26. Patient-Centered Outcomes Research Institute. PCORnet: The National Patient-Centered Clinical Research Network. Available from: http:// www.pcori.org/funding-opportunities/pcornet-national-patientcentered-clinical-research-network/. Accessed January 21, 2014.

27. Institute of Medicine. Digital Infrastructure for the Learning Health System - The Foundation for Continuous Improvement in Health and Health Care - Workshop Series Summary. Washington, DC, USA: National Academies Press; 2011. 
28. Institute of Medicine. Sharing Clinical Research Data: Workshop Summary. Washington, DC, USA: National Academies Press; 2013.

29. Ghitza UE, Gore-Langton RE, Lindblad R, Shide D, Subramaniam G, Tai B. Common data elements for substance use disorders in electronic health records: the NIDA Clinical Trials Network experience. Addiction. 2013;108:3-8.
30. Tunis SR, Stryer DB, Clancy CM. Practical clinical trials: increasing the value of clinical research for decision making in clinical and health policy. JAMA. 2003;290:1624-1632.

31. Clancy C, Collins FS. Patient-Centered Outcomes Research Institute: the intersection of science and health care. Sci Transl Med. 2010;2(37):37cm18.

\section{Publish your work in this journal}

Substance Abuse and Rehabilitation is an international, peer-reviewed, open access journal publishing original research, case reports, editorials, reviews and commentaries on all areas of addiction and substance abuse and options for treatment and rehabilitation. The manuscript management system is completely online and includes a very quick and fair peer-review system. Visit http://www.dovepress.com/testimonials.php to read real quotes from published authors. 